\title{
Separate Face and Body Selectivity on the Fusiform Gyrus
}

\author{
Rebecca F. Schwarzlose, ${ }^{1,2}$ Chris I. Baker, ${ }^{1,2}$ and Nancy Kanwisher ${ }^{1,2,3}$ \\ ${ }^{1}$ Department of Brain and Cognitive Sciences and ${ }^{2} \mathrm{McGovern}$ Institute for Brain Research, Massachusetts Institute of Technology, Cambridge, \\ Massachusetts 02139, and ${ }^{3}$ Athinoula A. Martinos Center for Biomedical Imaging, Charlestown, Massachusetts 02129
}

Recent reports of a high response to bodies in the fusiform face area (FFA) challenge the idea that the FFA is exclusively selective for face stimuli. We examined this claim by conducting a functional magnetic resonance imaging experiment at both standard $(3.125 \times 3.125 \times$ $4.0 \mathrm{~mm})$ and high resolution $(1.4 \times 1.4 \times 2.0 \mathrm{~mm})$. In both experiments, regions of interest (ROIs) were defined using data from blocked localizer runs. Within each ROI, we measured the mean peak response to a variety of stimulus types in independent data from a subsequent event-related experiment. Our localizer scans identified a fusiform body area (FBA), a body-selective region reported recently by Peelen and Downing (2005) that is anatomically distinct from the extrastriate body area. The FBA overlapped with and was adjacent to the FFA in all but two participants. Selectivity of the FFA to faces and FBA to bodies was stronger for the high-resolution scans, as expected from the reduction in partial volume effects. When new ROIs were constructed for the high-resolution experiment by omitting the voxels showing overlapping selectivity for both bodies and faces in the localizer scans, the resulting FFA* ROI showed no response above control objects for body stimuli, and the $\mathrm{FBA}^{\star} \mathrm{ROI}$ showed no response above control objects for face stimuli. These results demonstrate strong selectivities in distinct but adjacent regions in the fusiform gyrus for only faces in one region (the FFA*) and only bodies in the other (the $\mathrm{FBA}^{*}$.

Key words: fMRI; FFA; faces; bodies; fusiform; object

\section{Introduction}

Does the ventral visual pathway contain cortical regions that are selectively involved in processing just a single class of visual stimuli (Allison et al., 1994; Kanwisher et al., 1997), or are all regions of the ventral pathway instead involved in graded and overlapping representations of multiple stimulus classes (Haxby et al., 2001)? Faces have served as a key test case for this debate, based in part on the fact that the fusiform face area (FFA) is activated considerably more strongly by images of faces than by other object classes. In this study, we address an important challenge to the claimed face selectivity of the FFA that arises from recent reports that the FFA may also respond strongly to images of bodies.

Specifically, two studies have found responses in the FFA that were higher to headless bodies than to control objects, although lower to bodies than faces (Kanwisher et al., 1999; Peelen and Downing, 2005) [see also Cox et al. (2004) and Hadjikhani and de Gelder (2003) for responses to body stimuli in the FFA]. One study even found that the FFA response was not significantly lower to body parts than to faces (Spiridon et al., 2005). Peelen

Received June 24, 2005; revised 0ct. 12, 2005; accepted 0ct. 13, 2005.

This work was supported by National Institutes of Health Grants EY13455 and EY016231 (N.K.), National Center for Research Resources Grant P41RR14075, the Mind Institute, and the National Science Foundation Graduate Research Fellowship Program. We thank Galit Yovel and Alexander Rivest for comments on this manuscript. We thank Ken Kwong for originally suggesting that we scan at higher resolution with thin slices and for identifying and ensuring the availability of the pulse sequence, Thomas Benner for modifying the BOLD sequence to work with the eight-channel coil and for optimizing protocols, and Larry Wald for technical advice.

Correspondence should be addressed to Rebecca Frye Schwarzlose, Brain and Cognitive Sciences, Massachusetts Institute of Technology, 77 Massachusetts Avenue, Cambridge, MA 02139. E-mail: beccafs@mit.edu.

D01:10.1523/JNEUROSCI.2621-05.2005

Copyright $\odot 2005$ Society for Neuroscience ～0270-6474/05/2511055-05\$15.00/0 and Downing also reported a fusiform region that we call the "fusiform body area" (FBA), which is adjacent to and overlaps with the FFA and responds more strongly to headless bodies than to objects, but equally to headless bodies and faces. [Note that the FBA is located on the ventral surface of the brain, far from the extrastriate body area (EBA) (Downing et al., 2001), which is on the lateral surface of the temporal lobe.] Collectively, these findings suggest a graded and overlapping pattern of responses in the fusiform gyrus (Haxby et al., 2001) rather than a strict spatial segregation of responses to faces and bodies. Here, we used a scanning resolution higher than that of previous studies to test the hypothesis that the apparent dual selectivity of both the FFA and the FBA for both faces and bodies may result from blurring of the responses from two adjacent but distinct cortical regions, one selectively responsive only for faces and the other only for bodies.

To do this, we identified the FFA and FBA with a blocked localizer scan and then tested their response magnitudes to a variety of face, body, and assorted everyday object stimuli with an event-related design administered in the same participants and scan sessions. We conducted this study at standard resolution $(3.125 \times 3.125 \times 4.0 \mathrm{~mm})$ in experiment 1 and at a higher resolution $(1.4 \times 1.4 \times 2.0 \mathrm{~mm})$ in experiment 2 . Using these methods, we found that the response to faces and bodies on the fusiform gyrus could be clearly dissociated with higher-resolution imaging.

\section{Materials and Methods}

Stimuli and design. Participants performed both localizer scans [to identify regions of interest (ROIs)] and event-related scans to test the selectivity of the ROIs. They completed five runs of the localizer scan, each of which included three $16 \mathrm{~s}$ fixation periods and two $16 \mathrm{~s}$ blocks of five 
stimulus classes each (faces, headless bodies, scenes, assorted everyday objects, and scrambled versions of the everyday objects). The order of conditions was palindromic within a scan, and the serial position of each condition was counterbalanced within participants across runs. Within each block, participants viewed 20 images of a single stimulus class (300 $\mathrm{ms}$ per image, with a $500 \mathrm{~ms}$ interstimulus interval). Scrambled object stimuli were constructed by superimposing a grid over the objects and relocating the component squares randomly. As participants watched these stimuli, they performed a one-back task in which they were asked to make a key-press whenever an image was repeated consecutively. The images were jittered slightly in their location on the screen to preclude use of low-level transients in performing the one-back task. Participants completed six to seven runs of the event-related experiment in the same scan session. Each of the runs was composed of a quasirandom order of stimuli from the following four stimulus conditions: faces, headless bodies, body parts, and cars. A fifth condition of assorted everyday objects was added for the high-resolution scans in experiment 2. Other stimulus conditions that were included in the event-related design to test different hypotheses will not be discussed here. Each image moved either downward or to the left, and the participants' task was to identify the direction of motion of each stimulus by pressing one key to indicate movement to the left and another to indicate movement down. There were 15 images per stimulus category and 13 image presentations per stimulus category per run. Different images were used in the localizer and the event-related experiment.

Functional imaging. Participants were scanned on a 3.0 T Siemens (Erlangen, Germany) Trio scanner at the Martinos Center for Biomedical Imaging (Charlestown, MA). Images were acquired with a Siemens eight-channel phased-array head coil and gradient echo single-shot echo planar imaging sequence. For experiment 1 conducted at standard resolution, 28 slices covered the whole brain (dimensions, $3.125 \times 3.125 \times$ $4.0 \mathrm{~mm}$; interslice gap, $0.8 \mathrm{~mm}$; repetition time, $2 \mathrm{~s}$; echo time, $30 \mathrm{~ms}$ ). For experiment 2 conducted at higher resolution, 15-18 slices were oriented approximately perpendicular to the calcarine sulcus $(1.4 \times 1.4 \times$ $2.0 \mathrm{~mm}$; interslice gap, $0.4 \mathrm{~mm}$; repetition time, $2 \mathrm{~s}$; echo time, $33 \mathrm{~ms}$ ). For both experiments, high-resolution MPRAGE anatomical images were also acquired for each participant. Seven participants were scanned for experiment 1 , and 10 were scanned for experiment 2 . The data from one of the participants in experiment 2 were excluded from the analysis because of excessive head motion $(>6 \mathrm{~mm})$.

Data analysis was performed using Freesurfer and FS-FAST software (http://surfer.nmr.mgh.harvard.edu/). Before statistical analysis, images were motion corrected (Cox and Jesmanowicz, 1999) and smoothed for the localizer runs only ( $5 \mathrm{~mm}$ full width at half-maximum Gaussian kernel for experiment 1; $3 \mathrm{~mm}$ for experiment 2).

Our functional magnetic resonance imaging (fMRI) analyses focused on the right hemisphere, because previous work has shown that the FFA is larger and more consistent in the right hemisphere (Kanwisher et al., 1997) and because it is only the right FFA that has been claimed to be strongly category selective (Grill-Spector et al., 2004). Regions of interest were visualized on slices and defined individually for each participant using the blocked localizer scans (as described below); we then used fROI (http://froi.sourceforge.net/) to extract the time courses of response for the event-related experiments in each ROI (see Fig. 2). Critically, the data used to define the ROIs were independent of the data used to calculate the response magnitudes for each stimulus category in each ROI.

Cortical surfaces were reconstructed using Freesurfer for three of the participants in experiment 2 based on previous anatomical scans of those participants.

\section{Results \\ Experiment 1}

The results of our standard resolution scans replicated previous studies (Peelen and Downing, 2005; Spiridon et al., 2005). The localizer data were used to identify both a face-selective right FFA (using a contrast of faces $>$ objects) and a body-selective right FBA (using headless bodies $>$ objects) with a threshold for both contrasts of $p<0.0001$ uncorrected in individual participants. A right FFA was identified in every participant, and a replicable right FBA was found in five of the seven participants. One participant had no FBA and another had a very small FBA (eight voxels), which failed to replicate the body selectivity observed in the localizer scans in subsequent event-related scans; these two participants were excluded from additional FBA analyses. The FFA and FBA overlapped in all participants who showed both ROIs. The average size of the right FFA ROIs was $1.53 \mathrm{~cm}^{3}$. Of the five participants with a right FBA, the average size of the FBA ROI was $0.86 \mathrm{~cm}^{3}$, and the overlap averaged $0.45 \mathrm{~cm}^{3}$ per participant.

The time course of the response from the event-related runs for each stimulus category in the right FFA and right FBA ROIs (defined from the localizer scans) are shown in Figure 2, $a$ and $b$. In an ANOVA on the peak response magnitudes among the five participants that demonstrated both FFA and FBA ROIs, the interaction of stimulus condition by ROI did not reach significance $\left(F_{(2,3)}=5.8 ; p=0.09\right)$. Planned comparisons revealed that the response to headless bodies in the FFA was significantly greater than to cars $(p<0.005)$ while still significantly lower than to faces $(p<0.01)$. In contrast, in the FBA, the responses to images of both faces and headless bodies were significantly higher than to cars (both, $p<0.05$ ), whereas there was no difference in the degree of activation for face and headless body stimuli in this region $(p>0.8)$. These data replicate the results reported by Peelen and Downing (2005) at a similar scanning resolution $(3.75 \times 3.75 \times 5.0 \mathrm{~mm})$, indicating elevated responses to both faces and bodies in both the FFA and FBA.

To test whether the high FFA response we observed to bodies might be attributable to the inference of a face from the headless body stimuli (Cox et al., 2004), we compared the FFA response to headless bodies (where a face might be inferred) with its response to assorted body parts (where the inference of a face is unlikely). In the FFA, the response to body parts was greater than to cars $(p<0.005)$ and lower than to faces $(p<0.007)$, whereas there was no significant difference between the responses to body parts and headless bodies $(p>0.9)$. These results argue against the possibility that the high responses to body stimuli in the right FFA are attributable to the inference of a face.

\section{Experiment 2}

The second experiment was the same as experiment 1 , except that it was conducted at higher resolution (voxel size, $1.4 \times 1.4 \times 2.0$ $\mathrm{mm}$ ) and a second baseline condition of assorted everyday objects was added. We isolated the right FFA and FBA ROIs using the same methods described above for experiment 1 . Figure 1 shows examples of slices and surface plots with these ROIs at high resolution. Figure 2, $c$ and $d$, shows the time courses of the responses to each stimulus condition in the event-related runs for the high-resolution FFA and FBA ROIs.

An ANOVA on the magnitude of the peak response in the event-related experiments revealed a significant interaction of ROI by stimulus condition $\left(F_{(2,7)}=23.3 ; p<0.002\right)$. Planned comparisons confirmed, as expected, that the selectivity of the FFA and the FBA for their preferred categories (on the basis of which these regions were identified in the localizer scan) was replicated in the event-related scans: the FFA responded significantly more strongly to faces than to cars, objects, headless bodies, and body parts (all four, $p<0.005$ ), and the FBA responded significantly more strongly to headless bodies and body parts than to mixed objects and cars (all four, $p<0.005$ ).

Following up on the interaction of ROI by stimulus category, planned comparisons tested whether any selectivity for bodies could be found in the FFA and whether any selectivity for faces 


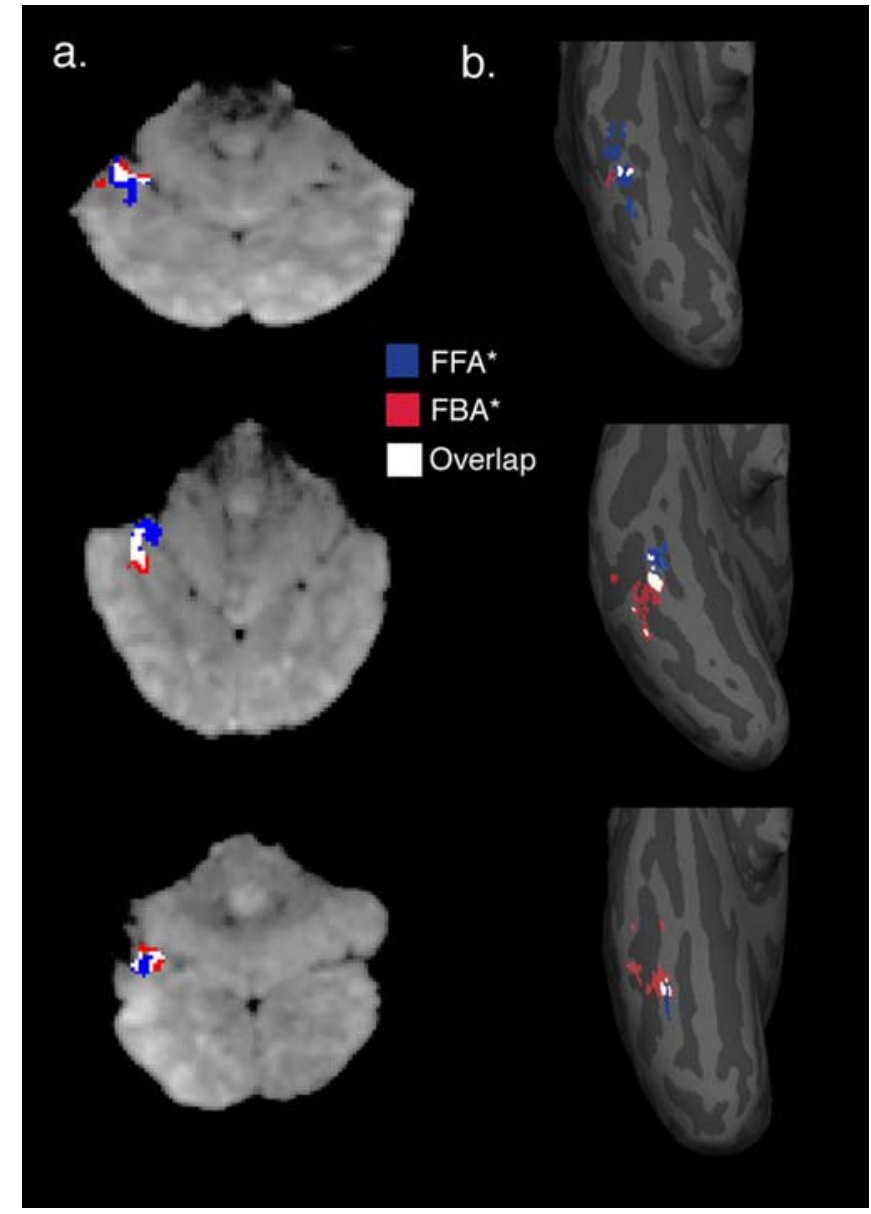

Figure 1. Examples of face and body ROls at high resolution. $\boldsymbol{a}$, Examples from three participants of FFA* (blue) and FBA* (red) ROls, as well as the overlap (white) between FFA (defined by faces $<$ objects) and FBA (defined by bodies $>$ objects). ROls are shown on functional image slices from three participants. The slices are left-right reversed, with posterior regions shown at the bottom of each image and the cerebellum at the top. $\boldsymbol{b}$, The same regions in the same three participants mapped to each participant's inflated cortical surface. The view shown here is of the ventral temporal surface of the posterior portion of the right hemisphere, with the lower tip of each inflated hemisphere representing the occipital pole. The FFA* (shown in blue), FBA* (shown in red), and the overlap (white) show considerable variation in their sizes and relative locations on the cortex.

could be found in the FBA. In the FFA, the response to headless bodies was not significantly greater than to cars or mixed objects, nor was it significantly different from the response to body parts (all, $p>0.20$ ). Although the response to body parts was not significantly different from that to cars $(p>0.10)$, it trended toward a higher response than to mixed objects ( $p<0.05$ uncorrected, a significance level that would not survive a correction for the four comparisons of body stimuli to control object stimuli).

In the FBA, the response to the headless body stimuli trended toward a higher response than to faces ( $p<0.05$, uncorrected), whereas the response to body parts did not significantly differ from that to faces $(p>0.30)$. Nonetheless, the FBA response to faces was still higher than to mixed objects $(p<0.01)$, although not significantly different from that to cars $(p=0.09)$.

Thus, the selectivity of the FFA and FBA ROIs are stronger at high resolution than standard resolution, a result supported by a significant triple interaction (among the participants who had both FFAs and FBAs) of ROI, stimulus type, and experiment $\left(F_{(2,11)}=5.07 ; p<0.05\right)$. This difference in selectivity might, at least in part, be attributable to the decrease in partial voluming

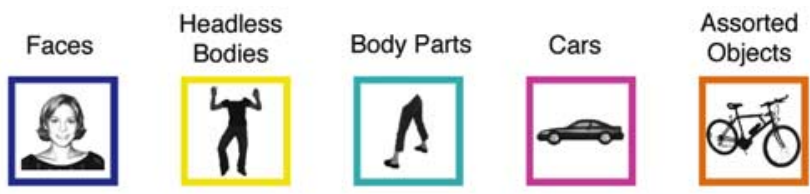

a. Standard Resolution FFA b. Standard Resolution FBA

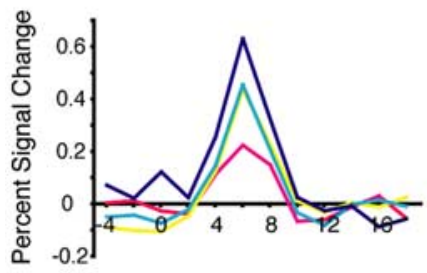

c. High Resolution FFA
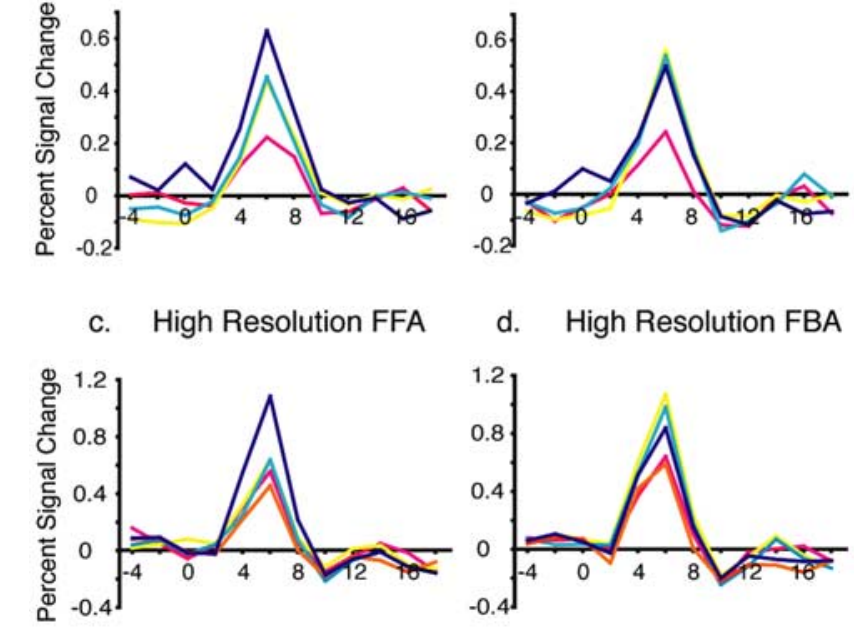

d. High Resolution FBA

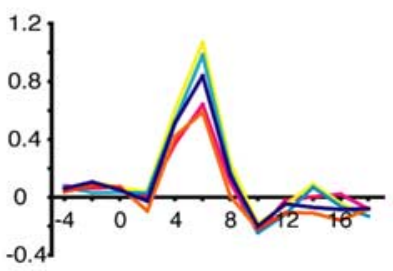

e. High Resolution FFA
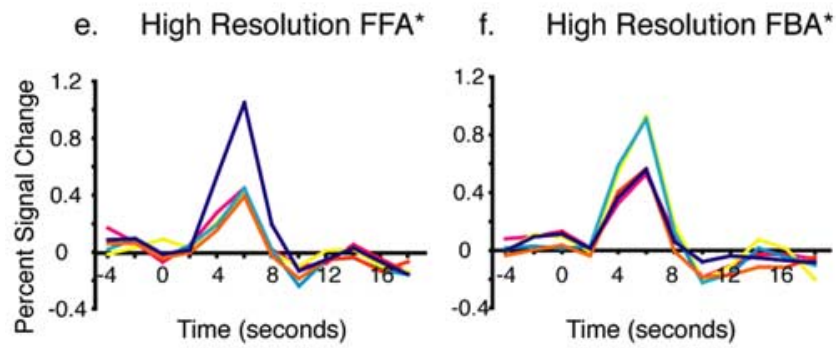

Figure 2. Time courses of the hemodynamic response in regions of interest. Examples of each of the stimulus conditions and their color code are shown across the top. Time courses of the hemodynamic response for each stimulus condition for the event-related runs averaged across participants are shown for the FFA $(\boldsymbol{a})$ and FBA $(\boldsymbol{b})$ from experiment 1 at standard resolution, as well as for the FFA $(\boldsymbol{c}), \operatorname{FBA}(\boldsymbol{d}), \mathrm{FFA}^{*}(\boldsymbol{e})$, and FBA* $(\boldsymbol{f})$ in experiment 2 at high resolution.

between face-selective and body-selective regions at high resolution. This potential explanation is supported by the reduced volume of the overlap between the FFA and FBA ROIs from standard to high resolution. Specifically, the average overlap at standard resolution constituted $0.45 \mathrm{~cm}^{3}$, or $27 \%$ of the total sum of the FFA and FBA ROIs, across the five standard resolution participants that demonstrated both ROIs, whereas the corresponding overlap was $0.17 \mathrm{~cm}^{3}$, or $18 \%$ of the summed FFA and FBA ROI volumes across high-resolution participants. However, despite this reduction of overlap at higher resolution, we still observed trends of higher responses to body parts than to control stimuli in the FFA and higher responses to faces than to control stimuli in the FBA.

We next attempted a stronger test of our hypothesis that the dual selectivity of the FFA and FBA may result from the pooling of responses from two adjacent but distinct cortical regions, one selective for only faces and the other selective for only bodies. To do this, we used a new ROI selection method in which we omitted from the right FFA ROI (which had an average of 162 voxels, or $0.64 \mathrm{~cm}^{3}$ ) all voxels that were also included in the FBA ROI to generate a new $\mathrm{FFA}^{\star} \mathrm{ROI}$ (mean 121 voxels, or $0.47 \mathrm{~cm}^{3}$ ), and we 
omitted from the right FBA ROI (mean 96 voxels, or $0.38 \mathrm{~cm}^{3}$ ) all voxels that were also included in the FFA ROI to generate a new $\mathrm{FBA}^{*} \mathrm{ROI}$ (mean 55 voxels, or $0.22 \mathrm{~cm}^{3}$ ). Importantly, as with our previous analyses, the blocked localizer data used to identify the $\mathrm{FFA}^{\star}$ and $\mathrm{FBA}^{\star}$ were independent from the event-related data we used to assess selectivity profiles in these ROIs (Fig. 2).

An ANOVA on the magnitude of the peak responses in the event-related data in these new ROIs revealed a strong interaction of ROI $\left(\mathrm{FFA}^{\star}\right.$ vs $\mathrm{FBA}^{\star}$ ) by stimulus condition (faces, headless bodies, and cars) $\left(F_{(2,7)}=31.4 ; p<0.001\right)$. As expected from previous analyses, the $\mathrm{FFA}^{\star}$ response was significantly higher to faces than to headless bodies, body parts, cars, and mixed objects (all four, $p<0.001$ ). More importantly, neither headless bodies nor body parts produced a higher response in the $\mathrm{FFA}^{\star}$ than did either cars or mixed objects (all four, $p>0.3$ ). Conversely, the $\mathrm{FBA}^{\star}$ responses to headless bodies and body parts were significantly greater than to faces, cars, and mixed objects (all six, $p<$ 0.02 ), whereas faces no longer produced a higher response than to cars or mixed objects (both, $p>0.4$ ). These results demonstrate selective responses (above control objects) only for faces in the $\mathrm{FFA}^{\star}$ and only for bodies in the FBA*.

To assess the relative locations of the $\mathrm{FFA}^{\star}$ and $\mathrm{FBA}^{\star}$ ROIs, we calculated the center of mass (COM) locations for these two ROIs in each individual high-resolution participant by taking the average of the in-slice row and column numbers in the matrix, as well as the slice number, across all voxels in each given ROI. As can be seen in the surface plots of Figure 1, we found a significant difference between the location of the right $\mathrm{FFA}^{*}$ and $\mathrm{FBA}^{\star} \mathrm{COMs}$ along the mediolateral axis, with $\mathrm{FFA}^{\star}$ medial to $\mathrm{FBA}^{\star}(p<$ $0.005)$. The average distance between these COMs was 2.2 voxels, or $3.1 \mathrm{~mm}$. Because our slices were oriented perpendicular to the calcarine sulcus and were therefore not aligned precisely from one participant to the next, it was difficult to accurately compare the ROI locations in the anteroposterior and dorsoventral dimensions between participants.

Finally, we addressed the question of whether exclusive selectivity on the fusiform gyrus could be demonstrated at standard resolution if the effects of partial voluming were minimized. We did this by selecting a single voxel in the FFA of each of our standard resolution participants that most reliably demonstrated a greater response to faces than to objects as measured by the $p$ value of this contrast in data from our blocked localizer runs. Crucially, the selection of these voxels was independent of their selectivity for bodies, and the pattern of response in these peak voxels was evaluated using our independent event-related data set. We found that the peak FFA voxels averaged across the standard resolution participants demonstrated a high response to faces $(1.08 \%$ signal change) with a response to headless bodies (0.43) and body parts $(0.52)$ that was no greater than to cars (0.41) ( $p>0.7$ and $p>0.3$, respectively). This finding demonstrates that exclusive selectivity on the fusiform gyrus can also be observed at standard resolution in circumstances for which the effects of partial voluming are minimized.

\section{Discussion}

In this study, we used high-resolution scanning techniques that uncovered a clear and striking dissociation between face and body selectivities on the fusiform gyrus. At a standard fMRI scanning resolution, face and body selectivity overlapped considerably, with substantial responses to body stimuli in regions identified as face selective and vice versa (Fig. $2 a, b$ ), as reported by Peelen and Downing (2005). However, at higher resolution, the observed selectivities become stronger, with responses to body stimuli in the FFA only slightly higher than to control objects (Fig. 2c). Finally, when new ROIs that omit regions of overlapping selectivity for faces and bodies were created, we found one region (the $\mathrm{FFA}^{*}$ ) that is selectively responsive only to faces, not bodies, and another region (the $\mathrm{FBA}^{*}$ ) that is selectively responsive only to bodies, not faces (Fig. $2 e, f$ ). These findings support our hypothesis that the dual selectivity of the FFA for both faces and bodies observed at standard resolution results from the pooling of responses from two adjacent but distinct cortical regions, one selective for only faces and another selective for only bodies.

In keeping with previous evidence from intracranial recordings (Allison et al., 1994), stimulation studies (Puce et al., 1999; Mundel et al., 2003) and neuropsychological studies (Wada and Yamamoto, 2001), our finding that some regions in the ventral visual pathway are apparently strongly selective for a single class of visual stimuli would seem to argue against the idea that all regions in the ventral visual pathway participate in the representation of each object (Haxby et al., 2001). However, two caveats must be mentioned here. First, the fact that strong and separate cortical selectivities exist for faces and bodies in the ventral visual pathway does not mean that the same will be found for all stimulus categories. Indeed, current evidence suggests that the cortical selectivities for faces and bodies may be unusual cases, contrasting with the more distributed and overlapping responses to multiple object categories in other cortical regions such as the lateral occipital complex (Malach et al., 1995). Second, although the $\mathrm{FFA}^{\star}$ and $\mathrm{FBA}^{\star}$ are uniquely selective for faces and bodies, respectively, compared with control stimuli (mixed objects and cars), both of these regions produce positive responses to nonpreferred stimuli compared with a fixation baseline. The role of these nonpreferred responses in the coding of objects is an important open question that is now being tested using a variety of neuroimaging methods (Haxby et al., 2001; Spiridon and Kanwisher, 2002; Grill-Spector et al., 2004). Currently, the strongest evidence that face-processing regions do not play an important role in the recognition of nonface objects comes from studies of neurological patients with very selective deficits in face recognition but not in general object recognition (Wada and Yamamoto, 2001).

The results of our study also have methodological relevance in highlighting the importance of scanning resolution when investigating functional segregation in the cortex [see also Beauchamp et al. (2004)]. Regions selectively responsive to faces and bodies that were clearly dissociable at high resolution were not dissociable at standard resolution. How can we determine how much resolution is enough to make such a distinction for any given study? The answer will depend on the grain of the cortical organization under investigation, with the response profiles of relatively large cortical regions such as the PPA less dependent on scanning resolution than smaller regions that may only be detected at high resolution. These considerations lead to an important asymmetry in the conclusions that can be drawn from fMRI studies: when clear functional dissociations are demonstrated between adjacent cortical regions, such results cannot be overturned by future studies at higher resolution, whereas any failure to find a functional dissociation [e.g., Shuman and Kanwisher (2004)] will always be contingent on the outcome of future studies at higher resolution. For example, the current results suggest that it will be necessary to revisit previous claims that the FFA may be responsive not only to faces but also to biological motion (Grossman and Blake, 2002), animations implying intentional agency (Schultz et al., 2003), visual expertise (Gauthier et al., 1999; Gauthier et al., 2000; Xu, 2005), and animals (Chao et al., 
1999). Indeed, it seems possible that many of these activations previously attributed to the FFA arise not from the FFA* but from the $\mathrm{FBA}^{\star}$ or another adjacent but distinct cortical region.

Beyond their methodological implications, the present results also raise a host of questions for future research. What is the function of the FBA, and how does it differ from that of the EBA? Given that lesions affecting the FFA are likely to also affect the FBA, do acquired prosopagnosic patients show deficits in body perception, and if so, in what aspects of body perception? More generally, why do face and body selectivities land nearby in the cortex, not only in the fusiform gyrus but also in lateral temporal cortex in both humans and monkeys (Tsao et al., 2003; Pinsk et al., 2005)?

Another question raised by our findings concerns the nature of the overlap region between the face and body selectivities on the fusiform gyrus. One previous study has suggested that the area of overlap between two functional regions might play a role in the integration of information processed by the neighboring regions (Beauchamp et al., 2004). Although this explanation is possible both for their case and ours, another possibility is that the observed dual selectivity reflects distinct but interleaved neural populations that perform no integrative function. Distinguishing between these hypotheses will require other methods such as fMRI adaptation.

In summary, our findings demonstrate two adjacent regions in the fusiform gyrus, one selectively responsive to bodies but not faces and an adjacent region selectively responsive to faces but not bodies. The striking dissociation in the category selectivity of these regions was not clear when standard scanning methods were used (Peelen and Downing, 2005), underlining the importance of resolution for investigations of functional specificity of the cortex.

\section{References}

Allison T, McCarthy G, Nobre A, Puce A, Belger A (1994) Human extrastriate cortex and the perception of faces, words, numbers and colors. Cereb Cortex 4:544-554.

Beauchamp MS, Argall BD, Bodurka J, Duyn JH, Martin A (2004) Unraveling multisensory integration: patchy organization within human STS multisensory cortex. Nat Neurosci 7:1190-1192.

Chao LL, Martin A, Haxby JV (1999) Are face-responsive regions selective only for faces? NeuroReport 10:2945-2950.

Cox D, Meyers E, Sinha P (2004) Contextually evoked object-specific responses in human visual cortex. Science 304:115-117.

Cox R, Jesmanowicz A (1999) Real-time 3D image registration for functional MRI. Magn Reson Med 42:1014-1018.

Downing PE, Jiang Y, Shuman M, Kanwisher N (2001) A cortical area selective for visual processing of the human body. Science 293:2470-2473.

Gauthier I, Tarr MJ, Anderson AW, Skudlarski P, Gore JC (1999) Activation of the middle fusiform "face area" increases with expertise in recognizing novel objects. Nat Neurosci 2:568-573.
Gauthier I, Skudlarski P, Gore JC, Anderson AW (2000) Expertise for cars and birds recruits brain areas involved in face recognition. Nat Neurosci 3:191-197.

Grill-Spector K, Knouf N, Kanwisher N (2004) Fusiform face area subserves face perception, not generic within-category identification. Nat Neurosci 7:555-562.

Grossman ED, Blake R (2002) Brain areas active during visual perception of biological motion. Neuron 35:1167-1175.

Hadjikhani N, de Gelder B (2003) Seeing fearful body expressions activated the fusiform cortex and amygdala. Curr Biol 13:2201-2205.

Haxby JV, Gobbini MI, Furey ML, Ishai A, Schouten JL, Pietrini P (2001) Distributed and overlapping representations of faces and objects in ventral temporal cortex. Science 293:2425-2430.

Kanwisher N, McDermott J, Chun MM (1997) The fusiform face area: a module in human extrastriate cortex specialized for face perception. J Neurosci 17:4302-4311.

Kanwisher N, Stanley D, Harris A (1999) The fusiform face area is selective for faces not animals. NeuroReport 10:183-187.

Malach R, Reppas JB, Benson RR, Kwong KK, Jiang H, Kennedy WA, Ledden PJ, Brady TJ, Rosen BR, Tootell RB (1995) Object-related activity revealed by functional magnetic resonance imaging in human occipital cortex. Proc Natl Acad Sci USA 92:8135-8139.

Mundel T, Milton JG, Dimitrov A, Wilson HW, Pelizzari C, Uftring S, Torres I, Erickson RK, Spire JP, Towle VL (2003) Transient inability to distinguish between faces: electrophysiologic studies. J Clin Neurophysiol 20:102-110.

Peelen MV, Downing PE (2005) Selectivity for the human body in the fusiform gyrus. J Neurophysiol 93:603-608.

Pinsk MA, DeSimone K, Moor T, Gross CG, Kastner S (2005) Representations of faces and body parts in macaque temporal cortex: a functional MRI study. Proc Natl Acad Sci USA 102:6996-7001.

Puce A, Allison T, McCarthy G (1999) Electrophysiological studies of human face perception. III. Effects of top-down processing on face-specific potentials. Cereb Cortex 9:445-458.

Schultz RT, Grelotti DJ, Klin A, Kleinman J, Van der Gaag C, Marois R, Skudlarski P (2003) The role of the fusiform face area in social cognition: implications for the pathobiology of autism. Philos Trans R Soc Lond B Biol Sci 358:415-427.

Shuman M, Kanwisher N (2004) Numerical magnitude in the human parietal lobe: tests of representational generality and domain specificity. Neuron 44:557-569.

Spiridon M, Kanwisher N (2002) How distributed is visual category information in human occipitotemporal cortex? An fMRI study. Neuron 35:1157-1165.

Spiridon M, Fischl B, Kanwisher N (2005) Location and selectivity of category-specific regions in human extrastriate cortex. Hum Brain Mapp, in press.

Tsao DY, Freiwald WA, Knutsen TA, Mandeville JB, Tootell RB (2003) Faces and objects in macaque cerebral cortex. Nat Neurosci 6:989-995.

Wada Y, Yamamoto T (2001) Selective impairment of facial recognition resulting from a haematoma restricted to the right fusiform and lateral occipital region. J Neurol Neurosurg Psychiatry 71:254-257.

$\mathrm{Xu} \mathrm{Y} \mathrm{(2005)} \mathrm{Revisiting} \mathrm{the} \mathrm{role} \mathrm{of} \mathrm{the} \mathrm{fusiform} \mathrm{face} \mathrm{area} \mathrm{in} \mathrm{visual} \mathrm{expertise.}$ Cereb Cortex 15:1234-1242. 